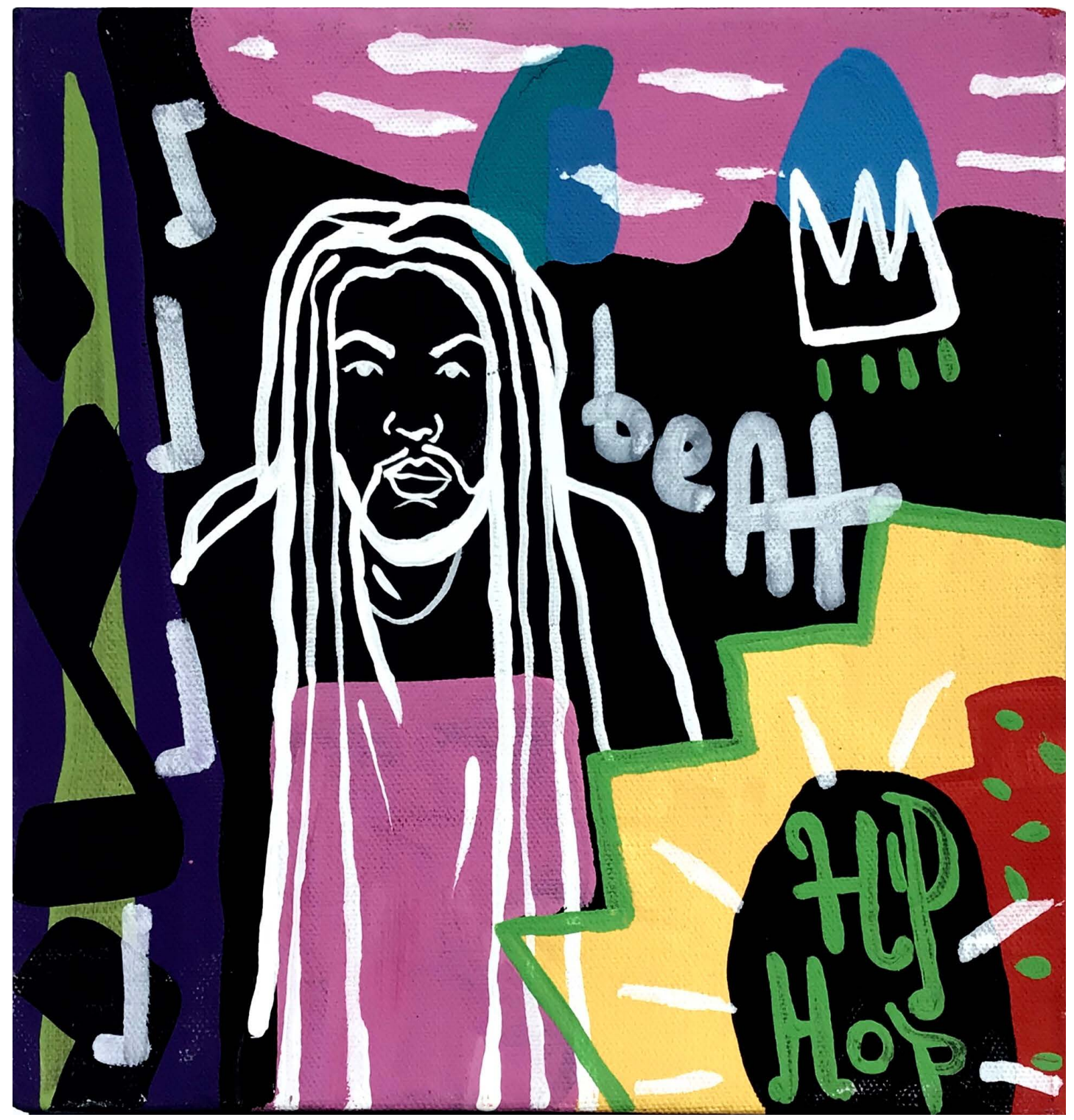

Artista invitado

Fabio Andrés Arboleda Mendoza

Beat

De la serie neWeed

Acrílico sobre lienzo

$20 \times 20 \mathrm{~cm}$

2020

Medellín 


\title{
Democracia, equilibrio informativo y pedagogía electoral. Análisis de la función orientadora del periódico regional La Patria en las elecciones locales de octubre de $201{ }^{*}$
}

\author{
Miguel Orlando Alguero Montaño (Colombia)**
}

\section{Resumen}

En los últimos años se ha observado que en las regiones de Colombia hay dificultad para acceder a los medios de comunicación, déficit de información y escasa formación democrática, y el cubrimiento periodístico se limita al registro noticioso de los hechos. Bajo este contexto, cobra importancia el papel de los medios regionales en el fortalecimiento de la democracia y de la libertad de expresión en las elecciones locales para elegir gobernadores, alcaldes, concejales, diputados y ediles. A partir de la agenda setting, el framing y la teoría funcionalista de la comunicación, esta investigación busca determinar si el periódico regional La Patria de Manizales, Caldas, cumplió su función orientadora en las elecciones locales de octubre de 2019. La metodología se basó en el enfoque cualitativo, se realizó un análisis de contenido de las publicaciones del diario, se aplicó una encuesta a lectores y suscriptores. Según los hallazgos, se concluyó que sí hubo pedagogía electoral por parte del periódico, el cual publicó contenidos con función orientadora, pero predominó más el enfoque logístico que el programático. Hubo elementos para que los lectores votaran de manera informada y se estableció una agenda periodística que priorizó el equilibrio informativo para que los candidatos tuvieran igual presencia en la información.

\section{Palabras clave}

Comunicación Política; Política Local; Elecciones; Medios de Comunicación Regionales; Pedagogía Electoral.

\footnotetext{
* Artículo derivado del proceso de investigación para optar al título de magíster en Estudios Políticos en la Universidad de Caldas, Colombia, El rol orientador del periódico regional La Patria en las elecciones locales del año 2019, y dirigido por el doctor Juan Carlos Yepes Ocampo. Se precisa que hay un conflicto interés del investigador debido a que laboró en el periódico La Patria durante la investigación, pero esta entidad no dio incentivos ni indicaciones en el proceso.

** Comunicador social-periodista. Periodista redactor del periódico La Patria, Colombia. Correo electrónico: miguelorlando89@gmail.com - Orcid: 0000-0003-0550-6783 - Google Scholar: https:// scholar.google.com/citations?user=DAE_P3cAAAAJ\&hl=es
} 
Cómo citar este artículo

Alguero Montaño, Miguel Orlando. (2021). Democracia, equilibrio informativo y pedagogía electoral. Análisis de la función orientadora del periódico regional La Patria en las elecciones locales de octubre de 2019. Estudios Políticos (Universidad de Antioquia), 62, pp. 241-265. https://doi.org/10.17533/udea.espo. n62a10

\title{
Democracy, Informative Balance, and Electoral Pedagogy. Analysis of the Guiding Function of the Regional Newspaper La Patria in the Local Elections of October 2019
}

\begin{abstract}
In recent years, it has been observed that in Colombia's regions there are difficulties in accessing the media, a deficit of information and little democratic training, and journalistic coverage is limited to the recording of news events. In this

[242] context, the role of the regional media in strengthening democracy and freedom of expression in local elections to elect governors, mayors, councilors, deputies and town councilors is important. Based on agenda setting, framing and functionalist communication theories, this research seeks to determine whether the regional newspaper La Patria of Manizales, Caldas, fulfilled its guiding role in the local elections of October 2019. The methodology was based on a qualitative approach, a content analysis of the newspaper's publications was conducted, a survey was applied to readers and subscribers. According to the findings, it was concluded that there was indeed electoral pedagogy on the part of the newspaper, which published contents with a guiding function, but the logistic approach was more predominant than the programmatic one. There were elements for readers to vote in an informed manner and a journalistic agenda was established that prioritized the informative balance so that the candidates had equal presence in the information.
\end{abstract}

\section{Keywords}

Political Communication; Local Politics; Elections; Regional Media; Electoral Pedagogy. 


\section{Introducción}

Los medios de comunicación antes, durante y después de las campañas electorales se presentan como actores políticos que inciden en la toma de decisiones colectivas a través de sus publicaciones. Su responsabilidad se basa en el equilibrio informativo, la pluralidad de voces y la veracidad de los hechos. Asimismo, las campañas electorales para las elecciones locales se convirtieron en un escenario adecuado para la materialización de la comunicación política. «Es el momento de mayor relación entre ciudadanos, políticos y medios de comunicación» (Restrepo, 2017). Olga Lucía Pérez (2014) agrega que las elecciones son el medio en el que se hacen efectivos los derechos de ser elegidos, elegir y fomentar la democracia.

En este panorama, candidatos y partidos políticos optan por ofrecer la información de sus propuestas o programas de gobierno, los ciudadanos despiertan el interés por conocer las promesas de los aspirantes y enterarse de la actividad electoral, y los medios de comunicación se esfuerzan por cubrir los hechos noticiosos políticos y asumir el rol de pedagogos electorales. Cuando el relacionamiento va de los medios a los ciudadanos, estos asumen un rol pedagógico, porque mediante la información explican el mensaje político (Mazzoleni, 2010).

Estos elementos son analizados por la Misión de Observación Electoral (MOE) en cada periodo electoral - tanto local como nacionalen Colombia. En una de sus publicaciones se evidencia que los medios, especialmente los periódicos, se quedan en el registro noticioso de las campañas y las elecciones y dejan a un lado su papel pedagógico u orientador, es decir, enseñarles a los ciudadanos cómo votar, presentarles y explicarles las propuestas de gobierno, y visibilizar las hojas de vida de los candidatos. Como falta pedagogía electoral, a los ciudadanos se les dificulta votar (MOE, 2016).

Dos trabajos del Grupo de Investigación en Periodismo (GIP) y del Grupo del Observatorio de Medios de la Facultad de Comunicación Social y Periodismo, de la Universidad de La Sabana, analizaron cómo funciona este rol orientador o pedagógico, especialmente en los medios impresos nacionales durante el cubrimiento de elecciones. Jairo Valderrama (2006), uno de los investigadores, concluyó que los medios cubrieron las 
elecciones desde la lógica de la noticia y dejaron a un lado el análisis profundo de los acontecimientos. Por su parte, el Grupo de Investigación Área de Periodismo (2002) analizó las campañas electorales 2002-2006 y encontró que el cubrimiento de la prensa se limitó al proceso electoral desde los candidatos y no desde sus programas de gobierno, basados en un enfoque informativo.

Bajo este contexto, ¿qué está pasando con los medios regionales?, ¿por qué hay problemas al momento de cubrir elecciones?, ¿por qué se dificulta poner en marcha el rol orientador durante las campañas electorales? Un estudio de la Fundación para la Libertad de Prensa (FLIP, 2019) sobre la situación del periodismo regional argumenta que las formas de hacer periodismo en las regiones están agrietadas. Este mismo estudio señala que en 353 municipios colombianos no hay presencia de algún medio de comunicación y que $29 \%$ de la población colombiana vive en municipios silenciados, es decir, los pobladores no tienen acceso a la información.

En octubre de 2019 se realizaron elecciones locales y fueron los medios regionales los que tuvieron el reto de explicarles a los ciudadanos los caminos complejos de los procesos electorales, y qué era conveniente [244] al elegir gobernadores, alcaldes, diputados, ediles y concejales, actores políticos que llevan las riendas de los departamentos y municipios.

Por consiguiente, esta investigación buscó responder al siguiente cuestionamiento: ¿cumple o no el periódico regional La Patria una función orientadora en los procesos electorales en el caso de las elecciones locales de 2019?

Los objetivos específicos que acompañaron el rumbo de la investigación fueron los siguientes: i) establecer el tipo de vínculos técnico-periodísticos que adopta el diario regional La Patria con los aspirantes a cargos de elección popular; ii) identificar los contenidos en el periódico regional La Patria que constituyan funciones orientadoras para los lectores; iii) detectar el influjo que, en materia de información, ejerce el periódico regional La Patria para que haya un voto informado por parte de los lectores.

Esta investigación tomó como referencias teóricas y bibliográficas lo desarrollado en la teoría funcionalista de la comunicación que se inició con 
Harold Lasswell (Leyva, 2001; McQuial, 2000; Klein, 2001), específicamente, lo expuesto sobre la función orientadora de los medios, que en el lenguaje moderno se conoce como pedagogía electoral (Consejo de Redacción, 2011, septiembre 29; MOE, 2016). También se tomaron las nociones de voto informado (Maigret, 2005; Gutiérrez, 2016; Angarita, 2017) y de equilibrio informativo (Rodríguez, 2010; Echeverría, 2017), contextualizado bajo los conceptos de elecciones regionales en Colombia (Barrero, 2016; Ronderos y Moreno, 2006; Luna, 2007) y medios regionales (Luna, 2007; Espinoza, 2004; Gómez, 2015; FLIP, 2019; Guerrero, 2010).

Otras teorías reseñadas en la investigación son la agenda setting o confección de la agenda mediática desarrollada por Maxwell McCombs en 1972 (Zunino, 2018; Rodríguez, 2004; Hernández, 2008; Valderrama, 2006; McQuial, 2000); y el framing o encuadre periodístico (Mazzoleni, 2010; Muñiz, Saldierna y Marañón, 2018; Entman, 1993). El trasfondo estuvo marcado por democracia y elecciones (Suárez, 2005; Sartori, 1991; Bobbio, 1997; Chavarri y Delgado, 2013; Mazzoleni, 2010), y la participación electoral (Nohlen, 2004; Torres, 2013; Medellín, 2014).

Asimismo, se evidenció que la comunidad académica ha despertado su interés por investigar cómo funcionan los medios de comunicación a partir de las ciencias políticas, cómo se relacionan con los actores políticos y la sociedad. Por ejemplo, Alejandra Barrios y Fabián Hernández (2012), en una investigación conformada por 15 universidades colombianas, monitorearon 34 medios de comunicación y a través del análisis de contenido indagaron sobre cómo era su cubrimiento. Por su parte, la MOE $(2016 ; 2018)$ realizó dos estudios en los que participaron alrededor de 24 universidades para analizar el cubrimiento informativo de medios de comunicación colombianos en dos momentos: el acuerdo de paz entre el Gobierno colombiano y las Fuerzas Armadas Revolucionarias de Colombia-Ejército del Pueblo (FARC-EP) y las elecciones presidenciales de 2018.

La novedad de esta investigación radica en que en Colombia no se ha examinado de forma detallada el rol orientador de los periódicos regionales, lo que se encuentra en la literatura científica son trabajos sobre medios nacionales y su cubrimiento durante las elecciones presidenciales. Por lo tanto, este artículo busca aterrizar en la esfera local lo que ya se ha hecho en la nacional, centrándose en las elecciones regionales. 


\section{Metodología}

Esta investigación tiene un enfoque cualitativo con alcance descriptivo (Hernández, 2014, p. 92). La unidad de análisis fue el periódico regional La Patria, que cubre al departamento de Caldas y su capital, Manizales. Hay que aclarar que cuando se precisaron los objetivos y alcance se pensó en abarcar la totalidad de Colombia, para tener varias miradas de cómo se da la función orientadora en diferentes regiones; sin embargo, en la indagación y solicitud de permisos de medios impresos regionales, el investigador se encontró con restricciones para acceder a la información contenida en bases de datos de suscriptores, debido a la protección de la Ley de Habeas Data (Ley Estatutaria 1581 del 17 de octubre de 2012). Eso llevó a tomar la decisión de solamente aplicar los instrumentos al periódico La Patria, donde sí hubo permisos. Para compensar esas limitaciones, se decidió ampliar el lapso de muestras de notas periodísticas de dos meses a cuatro. En cuanto al muestreo, se seleccionó el tipo de muestra no probabilística, intencionada o por conveniencia (Hernández, 2014, pp. 189 y 390).

Los criterios de selección fueron la accesibilidad y apertura a la consulta de información por parte de los suscriptores, para eso se determinó que

[246] fueran lectores frecuentes del diario, conocieran los contenidos publicados y tuvieran acceso tanto al periódico impreso como a la versión digital. Se aplicaron 100 encuestas, la caracterización arrojó 52 mujeres y 48 hombres, $55 \%$ pertenece al rango de edad entre 25 y 50 años, el nivel de escolaridad osciló entre pregrado y posgrado, $66 \%$ son suscriptores del diario y $34 \%$ lectores habituales o frecuentes. Se aplicaron 23 encuestas de manera digital, a través del formato de Google para encuestas, y 77 se realizaron de forma física a los suscriptores o lectores que se acercaron al periódico, a unos se les llevó hasta sus casas y a otros se les entregó en un lugar que indicaron.

Otro criterio de selección fue la temporalidad y la temática electoral para las notas periodísticas. Se escogieron las publicaciones desde el 27 de julio - fecha de cierre de inscripciones de los candidatos- hasta el 27 de noviembre - un mes después de las elecciones- relacionadas con la campaña electoral de 2019, para un total de cuatro meses, y así aplicar la matriz o rejilla de análisis. En total, fueron 222 noticias analizadas con base en las investigaciones realizadas por los grupos de investigación de la Universidad de La Sabana (Valderrama, 2006) y del Grupo de Investigación Área de Periodismo (2002). Se estipularon siete categorías: 
i) Géneros periodísticos: es la forma en que se presenta la información al público. Las subcategorías son: informativo o noticia, reportaje, crónica, de opinión, breves, informe especial y editorial.

ii) Contexto: los acontecimientos noticiosos no aparecen solos ni de la nada, tienen causas y derivación de otros hechos o situaciones. Las subcategorías son: antecedentes, consecuencias, trayectoria del personaje, propuestas del candidato, posiciones contrarias a esas propuestas y enfoque.

iii) Protagonistas: son aquellos que representan el protagonismo de las notas periodísticas. Las subcategorías son: candidato, partido, seguidores, proyecto o programa del candidato, funcionarios de la campaña y familiares del candidato.

iv) Fuentes: es de dónde proviene la información. Las subcategorías son: candidatos, miembros de la campaña, partido del aspirante, familiares, gobierno, sector privado, instituciones educativas, politólogo, investigador o experto, otro medio de comunicación o agencia de noticias, y población civil.

v) Interés adjudicado: es la intensidad y relevancia, en el periodismo se llama cobertura o despliegue. Para esta categoría se tuvo en cuenta el índice de atención de Richard Budd: «La Escala de Atención estableció cuatro elementos indispensables para este tipo de análisis (tamaño, espacio, lugar en la página y número de página). Que en estos casos busca demostrar el máximo interés que pudo alcanzar el texto periodístico en el medio» (Pinzón, Ocampo y Gutiérrez, 2015). A través de esta medición, Budd da una calificación binaria de 0 y 1 a seis variables desde una escala de seis puntos. La adaptación de esta escala para los periódicos o medios impresos se basa en tamaño, espacio, lugar de la página, número de página, acompañamiento gráfico y sumario.

vi) Autoría de las notas: se refiere a la firma de las notas, es decir, el nombre de quien escribe la noticia. Las subcategorías son: funcionario de la campaña, candidato, agencia de noticias, periodista del medio, corresponsal, enviado especial, columnista invitado y sin firma.

vii) Temática electoral: consiste en las actividades que se realizan antes, durante y después de la campaña electoral. Las subcategorías son: logística electoral, planes de gobierno, perfiles de los candidatos, delitos electorales, actividades de campaña —como inscripción, apertura y cierre-, pedagogía electoral, encuestas y debates. 
Para lograr los objetivos propuestos se aplicaron las siguientes técnicas e instrumentos: una entrevista semiestructurada (Hernández, 2014, p. 403) efectuada al jefe de Redacción del periódico, con un cuestionario de diez preguntas (véase anexo 1). La segunda es el análisis de contenido. Según Klaus Krippendorff (1990), el análisis de contenido «es una técnica de investigación destinada a formular, a partir de ciertos datos, inferencias reproducibles y válidas que puedan aplicarse a su contexto» (p. 28). El mismo autor agrega que se establecen unidades de análisis, que para esta investigación fueron las noticias publicadas por La Patria, las cuales se analizan de acuerdo con las categorías expuestas anteriormente.

La tercera técnica para esta investigación fue una encuesta diseñada a partir de un cuestionario de quince preguntas cerradas para los lectores o suscriptores del periódico, que pretende conocer qué tan importante es el cubrimiento electoral del periódico y si se encontró en el periódico noticias que los orientaran, como se expuso en anteriores párrafos (véase anexo 2). Por último, se realizó un cuestionario de cinco preguntas para entrevistar a cinco expertos en temas electorales, de periodismo, medios de comunicación y política. Se les preguntó sobre el equilibrio informativo, los elementos periodísticos para cubrir las elecciones, la importancia de la pedagogía electoral y los temas para orientar a los lectores (véase anexo 3).

\section{Resultados y discusión}

Esta investigación arrojó como resultado seis grandes hallazgos que comprenden aspectos como la pedagogía electoral, la confección de la agenda periodística, el equilibrio informativo, el encuadre de las noticias, el voto informado y la relación entre el periódico y los candidatos.

\subsection{Generalidades}

Se analizaron 222 publicaciones entre julio y noviembre de 2019, siendo octubre y septiembre los meses con más notas publicadas, ya que estos meses corresponden al centro de la temporada electoral (véase gráfica 1; véase anexo 4).

Las noticias políticas en el periódico La Patria se publican en la sección Público. Según la tabulación de los datos, esta fue la sección con más notas (139). Esto demuestra que el diario mantuvo un orden en sus publicaciones, garantizando que el lector encontrara los hechos políticos en las páginas que correspondían (véase gráfica 2). 
Gráfica 1. Publicaciones analizadas.

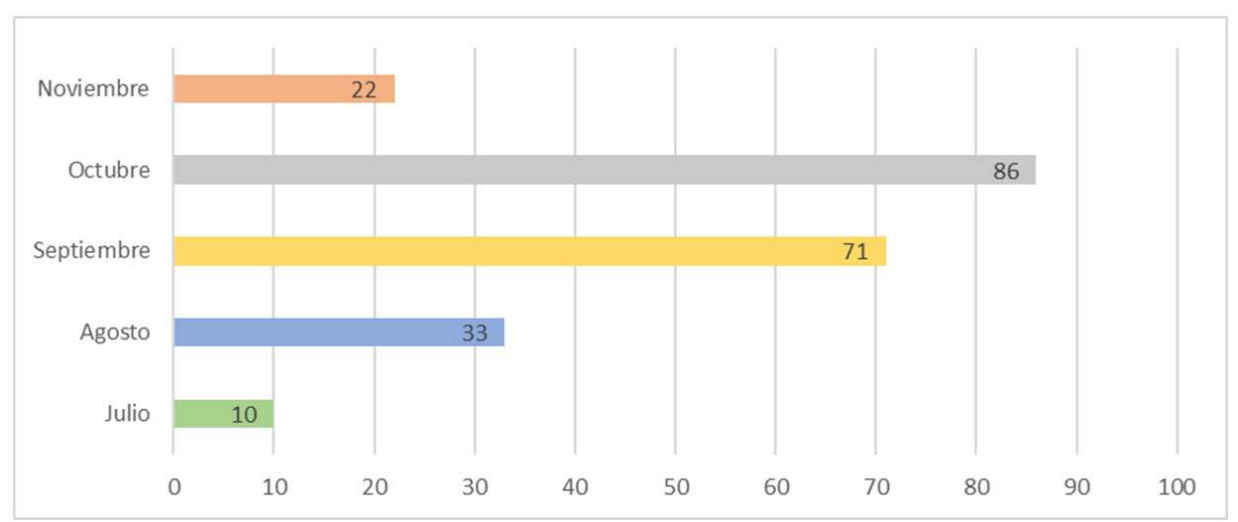

Fuente: elaboración propia.

Gráfica 2. Secciones del periódico, según las publicaciones analizadas.

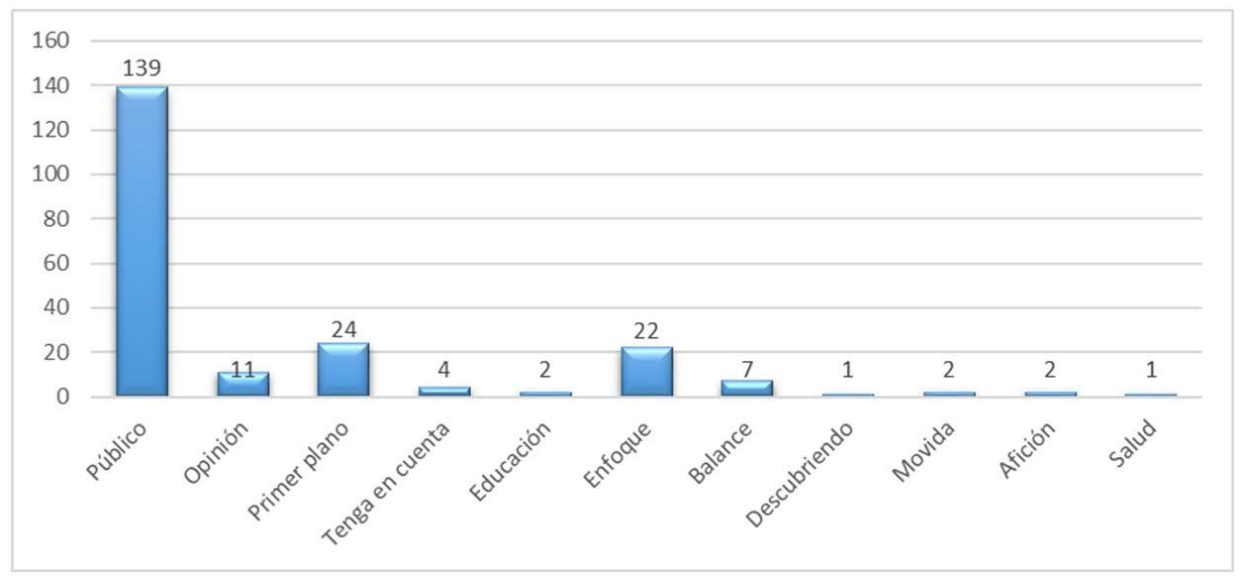

Fuente: elaboración propia.

\subsection{Temáticas, protagonistas y agenda}

La proposición principal de la agenda setting es que los medios poseen la capacidad de escoger y destacar los temas que consideran pertinentes y omitir otros que no son relevantes para la sociedad, por lo tanto, construyen una agenda que enfatiza ciertas noticias, que luego se convierten en rutas de diálogos para la opinión pública. Una agenda mediática es una jerarquía de prioridades o una lista de asuntos clasificados por importancia (Maigret, 2005). 
A partir del análisis de contenido, se halló que el tema que tuvo mayor presencia fue el de las actividades de campaña con $22 \%$, hechos que se generaron alrededor de los candidatos en sus actividades diarias. Esto muestra la intención del periódico de registrar el día a día de la contienda política. El resto de la torta está casi repartida por partes iguales en temas, pero resaltan los perfiles de los candidatos (14\%), planes de gobierno (13\%) y resultados electorales (11\%) (véase gráfica 3$)$.

Gráfica 3. Temática electoral.

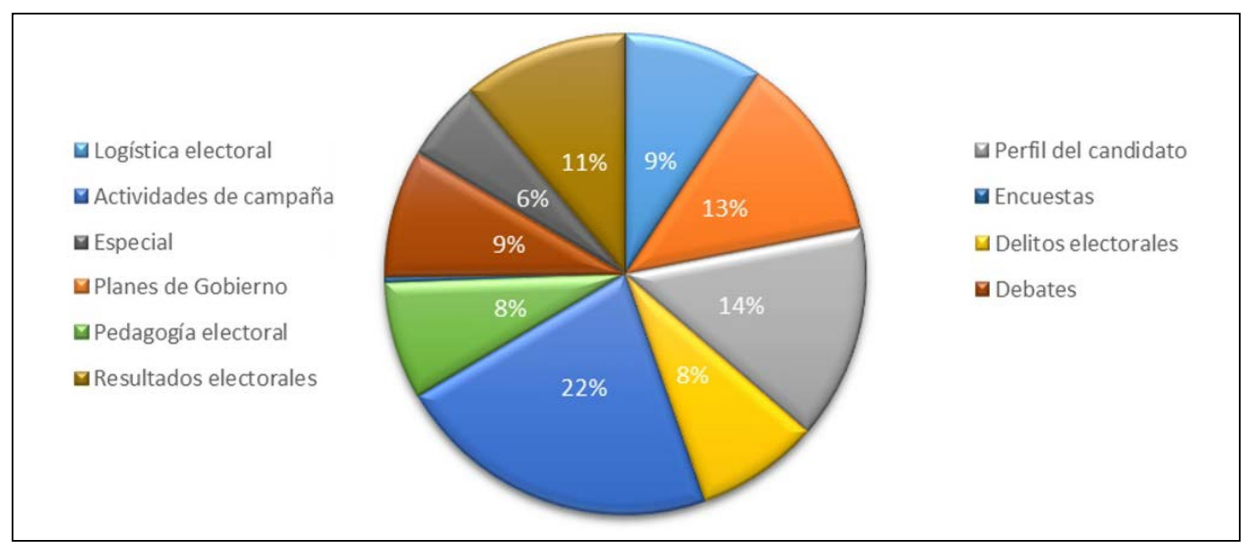

Fuente: elaboración propia.

Para completar estos resultados, si se mira en la encuesta a los lectores, estos temas fueron los preferidos por ellos. En la pregunta 12 se les solicitó señalar la temática electoral de su interés, ante lo cual mencionaron los resultados electorales, las propuestas de los candidatos y cómo votar, temas relacionados directamente con la pedagogía electoral. En esta misma línea, el jefe de Redacción, en la pregunta 5 de la entrevista, señaló que los perfiles de los candidatos y los planes de gobierno estuvieron en el centro de la cobertura, al igual que los temas pedagógicos para enseñarles a los lectores las novedades de esta elección.

Se hizo una tarea de consultar a todos los candidatos a las alcaldías, presentar sus perfiles, sus biografías, sus propuestas principales, hacerles una pregunta en torno a su idea de gobernar, y se hicieron algunos trabajos de agenda propia, más enfocados tanto en el análisis en cómo se estaba moviendo el tema político del departamento, y a temas pedagógicos para enseñarle a la gente (Jefe de Redacción, comunicación personal, noviembre 21, 2019). 
De igual forma, los cinco expertos consultados para la investigación mencionaron como temas las preguntas relacionadas con la organización electoral, los perfiles de los candidatos, las promesas o propuestas de campaña, los programas o planes de gobierno, alianzas políticas, financiadores de campañas, los riesgos y alertas en las elecciones.

Las preguntas frecuentes relacionadas con la organización electoral. También las hojas de vida de los candidatos, sus antecedentes, promesas de campaña, programas de gobierno. Esto se debe acompañar con información relacionada con el entorno político y cómo se da la participación de minorías (Experto 1, comunicación personal, abril 28, 2020).

Las propuestas de candidatos para visibilizar lo que es posible y lo que no es, eso ayudaría a los ciudadanos a razonar acerca de quiénes les están haciendo promesas que no tienen posibilidad de ser cumplidas (Experto 2, comunicación personal, mayo 5, 2020).

Lo programático debe ser lo primero. Es decir, insistir en el análisis, la verificación, la confrontación y averiguación de los programas de gobierno o propuestas de los candidatos (Experto 3, comunicación personal, mayo 2, 2020).

Temas de la organización electoral en sí, otros de las campañas y su logística (Experto 4, comunicación personal, abril 30, 2020).

Un tema central es si el plan de gobierno de cada candidato se puede cumplir cuando se plasme en su plan de desarrollo (Experto 5, comunicación personal, mayo 8, 2020).

Luego de estas temáticas, se presentó la pregunta ¿quién es el protagonista de esos temas? En el análisis de las notas publicadas se encontró que la mitad de las publicaciones corresponden a los candidatos como protagonistas del hecho noticioso: $20 \%$ hace referencia a las actividades electorales durante la campaña política y $11 \%$ a los programas o propuestas de los candidatos. En general, los protagonistas de las notas publicadas fueron los candidatos, sus propuestas y actividades. Esto comprueba una apuesta por el periódico para presentar los programas de gobierno de los candidatos, pero al mismo tiempo registrar el día a día del proceso electoral (véase gráfica 4).

De esta manera, al igual que la agenda setting, la teoría del framing explica cómo los medios de comunicación construyen sus contenidos, a qué temas le dan más importancia y cómo los muestran. ¿Por qué el framing es 
relevante durante las campañas electorales? Porque los medios desempeñan un papel crucial en las elecciones, ya que, al efectuar su propio tratamiento informativo, a la hora de presentar los temas de campaña, los candidatos y sus propuestas permiten que los electores y ciudadanos tengan acceso al debate político.

Gráfica 4. Protagonista de las publicaciones.

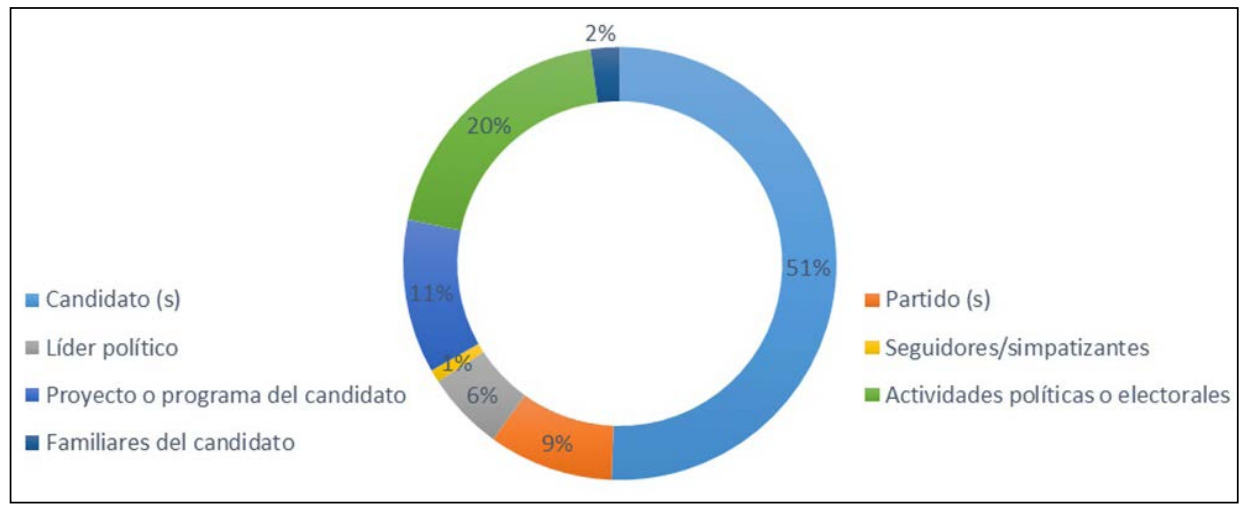

Fuente: elaboración propia.

Continuando con los resultados sobre cómo se hizo, un punto es la agenda que planteó el diario. En la entrevista al jefe de Redacción se mencionó en las respuestas 5 y 11 que el periódico tuvo una agenda propia con base en un plan para la cobertura que tenía como objetivo plantear temas para ayudar a entender las propuestas de los candidatos. Esta agenda se complementó con informes de análisis, temas pedagógicos sobre las novedades de las elecciones, planes de gobierno y votación. «Trazamos un plan que implique la mayor cobertura posible con una agenda propia muy fuerte, plantear temas de verdad que ayuden a entender las propuestas de los candidatos» (Jefe de Redacción, comunicación personal, noviembre 21, 2019).

\subsection{Interés, relevancia y equilibrio}

Para este punto se usó el índice de atención de Richard Budd (Pinzón, Ocampo y Gutiérrez, 2015). Este indicador aplicado a medios impresos lo que busca es demostrar cómo ese periódico usó la imagen, el espacio, la diagramación, el texto y el orden para darle mayor o menor relevancia 
a las publicaciones de su cubrimiento periodístico. La mitad de sus notas estuvieron entre el máximo de interés y el interés alto, incluso parte del otro $50 \%$ se ubicaron entre interés medio alto e interés medio (véase gráfica 5). Se demuestra, por tanto, que el diario les sumó importancia y relevancia a sus notas para suscitar interés en el lector, es decir, las publicaciones del diario mantuvieron elementos para generar mayor impacto y así captar la atención a través de la imagen, la palabra, el espacio y el orden.

Gráfica 5. Interés adjudicado por el periódico.

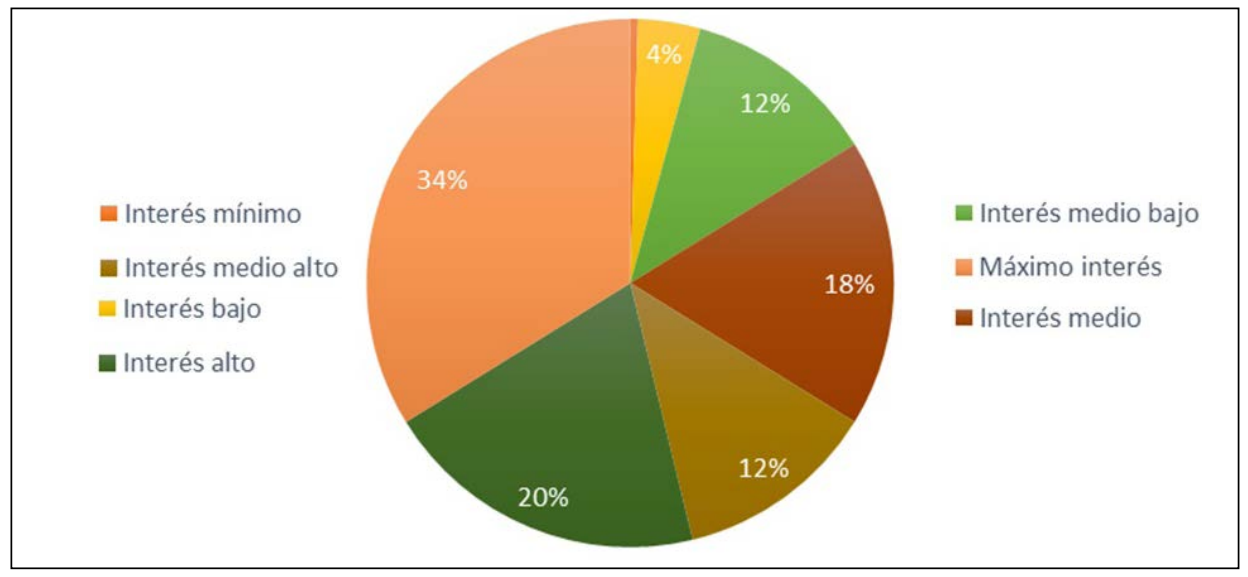

Fuente: elaboración propia.

Esas cifras dan pie para preguntar: ¿esa relevancia adjudicada por el periódico a sus notas generó algún interés en sus lectores? Si se revisa la pregunta 8 de la encuesta a los lectores sobre cómo calificaron el cubrimiento electoral, $90 \%$ respondió que entre muy importante y extremadamente importante fue el cubrimiento electoral, lo que manifiesta tanto la preponderancia de las notas como la relevancia que les dieron los lectores a esas notas. A esto se suma el tipo de preferencias de los lectores hacia temas locales y de política, así se evidenció en la cuarta pregunta de la encuesta, en la que se les indagó sobre la consulta de secciones del periódico. También se encontró que el papel del periodismo no consiste solamente en garantizar la equidad y el equilibrio, sino también en dar elementos de análisis. «Es necesario orientar a los receptores de dicha información, a fin de lograr que la elección que hagan en las mesas de votación sea la más adecuada para salvaguardar los intereses del país y de sus ciudadanos» (Grupo de Investigación Área de Periodismo, 2002, p. 4). 
Frente a esto, se recomienda un cubrimiento exhaustivo y proporcionado, guardando el equilibrio informativo para fortalecer la democracia, ya que al proporcionarles a los lectores información suficiente, de calidad, rigurosa y equitativa de cada candidato, tendrán elementos suficientes para tomar una decisión ante las urnas (Kovach y Rosenstiel, 2003). A su vez, este equilibrio informativo es la presentación de información equitativa, bien sea en la elección y uso de fuentes o enfoques para validar los hechos durante las campañas electorales, o el acceso equilibrado que brinda el medio para que los candidatos estén en igualdad de condiciones en los espacios de publicación (Rodríguez, 2010).

\subsection{Aspectos formales y técnicos}

Respecto a esta categoría, se evidenció que el género periodístico más usado fue el informativo o noticioso $(45 \%)$, esto hace alusión a la forma básica de presentar los hechos noticiosos, respondiendo a las preguntas qué, cómo, cuándo, dónde, por qué y para qué. También es el más sencillo para contar lo que ocurre en el ambiente político. Le siguen los informes especiales durante la temporada electoral (39\%), en los que se destacan los perfiles de los candidatos, los análisis de sus planes de gobierno, las cifras de

[254] los resultados electorales y los análisis del panorama político de Manizales y de Caldas (véase gráfica 6).

Gráfica 6. Géneros periodísticos.

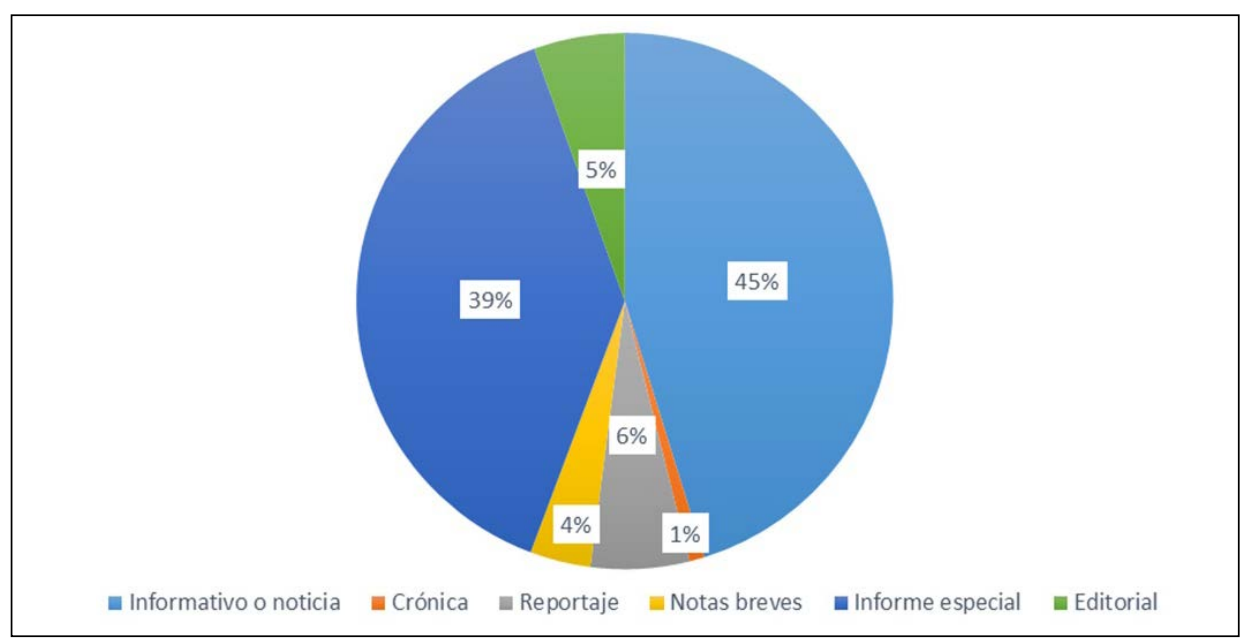

Fuente: elaboración propia. 
Otros elementos formales son las fuentes (véase gráfica 7) y los recursos de contexto en las notas (véase gráfica 8). La variedad primó en estas categorías. Las voces y fuentes de información pasaron por los candidatos, entidades como la Registraduría Nacional del Estado Civil, el Consejo Nacional Electoral y la Misión de Observación Electoral, hasta expertos en temas políticos y electorales. Esto se enriqueció con otras herramientas que se usan en las notas como recuadros explicativos e información de contexto como consecuencias y efectos del hecho noticioso, y voces a favor o en contra de las propuestas de los candidatos. La finalidad de estos elementos es darle veracidad, precisión, calidad y pluralidad de información a las notas publicadas.

Gráfica 7. Fuentes consultadas.

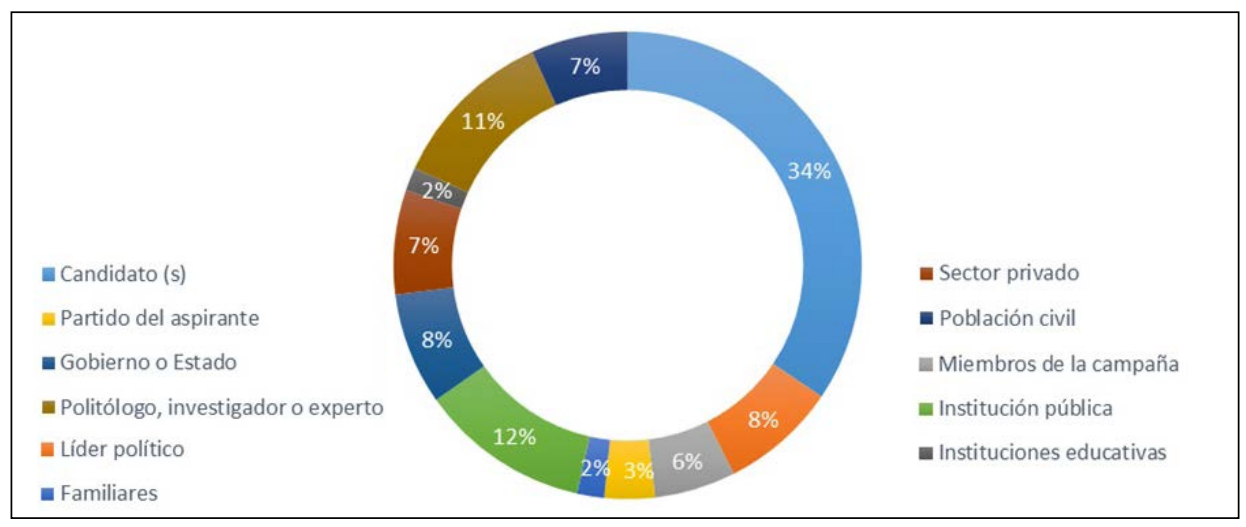

Fuente: elaboración propia.

Teniendo en cuenta estas dos categorías, en la encuesta a los lectores se les preguntó sobre qué tan precisos fueron los medios o canales de comunicación a la hora de informar y cubrir las elecciones. Los resultados de la séptima pregunta revelaron que el periódico La Patria fue el medio más preciso en sus publicaciones, estando por encima de otros medios como radio y televisión. En esta misma línea entra el elemento equilibrio informativo, como se dijo anteriormente. En la entrevista se anotó en la pregunta 6 que este equilibrio tiene que ver con la ponderación de las fuentes, de los candidatos y de los hechos noticiosos. 
Gráfica 8. Contexto en las notas publicadas.

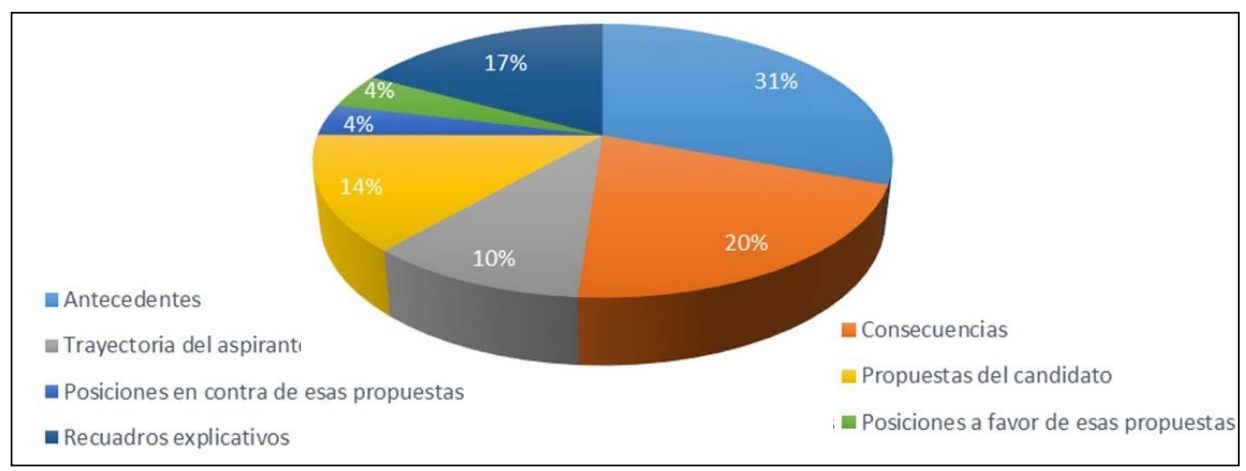

Fuente: elaboración propia.

\subsection{Tendencia e ideología}

Lo contrario al equilibrio, precisión y claridad es el sesgo informativo, que en muchas ocasiones está determinado por las afinidades que hay entre el medio y alguna tendencia política. Se considera una violación a las reglas básicas de objetividad e imparcialidad periodística. «En el ámbito del periodismo político se entiende por sesgo la tendencia sistemática a

[256 ] beneficiar a unos actores o posiciones en detrimento de otros» (Echeverría, 2017, p. 219). De esta manera, la agenda informativa o mediática se debate entre el equilibrio informativo y el sesgo periodístico.

En la entrevista se le preguntó al jefe Redacción (comunicación personal, noviembre 21,2019 ) si es importante que un medio regional cubra las elecciones locales sin ningún sesgo político, a lo que respondió que cubrir sin sesgos es generar credibilidad entre los ciudadanos. En la misma entrevista, se le interrogó si la ideología política cuando se fundó el periódico se mantuvo o cambió, a lo que aseveró que como los medios se modernizaron lo hicieron también sus relaciones con los partidos políticos. En la pregunta 4 insistió en que el periódico no tiene ningún vínculo o cercanía con algún partido que haya afectado el cubrimiento electoral y se recurrió a la ecuanimidad en las condiciones para las publicaciones en el periódico. Obviamente, esta perspectiva se ubica en un plano que puede registrar algunos niveles de subjetividad.

Frente a este tema, a los lectores se les preguntó si estaban de acuerdo o no con que el periódico tuviera relación editorial con alguna tendencia 
política. Más de la mitad (57\%) respondieron que estaban totalmente desacuerdo y en desacuerdo, y $23 \%$ que ni de acuerdo ni en desacuerdo. Esto evidencia un interés de los lectores de que no haya alguna relación o identificación entre el diario y un partido político, es decir, que se mantenga al margen, lo que va en la misma línea del propósito del periódico de no mantener algún vínculo con partido o ideología política.

\subsection{Rol orientador}

La función orientadora es la parte central de esta investigación. La pedagogía electoral, como también es conocida, busca dar elementos de análisis a los lectores para que tengan herramientas e información necesaria para tomar decisiones. «Es importante y necesaria en la información política, para que el público no se quede en la simple recepción de las noticias, sino que obtenga un criterio fundamentado acerca de las diversas actividades sociales» (Klein, 2001). Partiendo de esto, las categorías de análisis mostraron qué contenidos se publicaron y cómo se llevó a cabo esa función en el periódico; mientras que en la encuesta a los lectores se les indagó si las notas publicadas por el periódico los orientaron durante el proceso electoral. El $79 \%$ indicó que entre mucho y demasiado, un porcentaje alto que muestra la favorabilidad de los lectores hacia esta función del medio.

Al preguntarle al jefe de Redacción (comunicación personal, noviembre 21, 2019) sobre cómo se desarrolló ese rol o función orientadora en el proceso electoral, afirmó que se centró en la explicación acerca de qué es el umbral, la doble militancia, lo que implica un programa de gobierno, qué es el voto programático, cómo se distribuye el presupuesto de la Alcaldía y cómo reciben los alcaldes sus municipios. Aseguró que en esa función se trata de orientar a los ciudadanos sobre sus derechos y deberes frente a las elecciones.

En las preguntas 10 y 16, el jefe de Redacción (comunicación personal, noviembre 21,2019) expresó que explicarles a los ciudadanos cómo y por qué se vota es una herramienta importante para la democracia, tanto como la participación para hacer veeduría a los programas de gobierno. Recalcó que esto fue un espacio abierto para que los lectores votaron informados, esa es la relevancia de la pedagogía electoral.

Se define el voto informado como un voto razonado y sensato, ya que se asume con responsabilidad el derecho de sufragar. 
Informarse lo mejor posible acerca de qué o a quién se elige y para qué es una responsabilidad ciudadana que da fuerza al voto. Esto plantea la necesidad de escuchar objetivamente las propuestas de los distintos candidatos o a los postulantes de las diferentes opciones que se nos proponen y no solo a nuestro favorito sentimental (Gutiérrez, 2016, p. 22).

Se les preguntó a los cinco expertos si consideran que esa pedagogía electoral efectuada por un medio regional es una herramienta para fortalecer la democracia. Aseguraron que esta mantiene la libertad de expresión y garantiza la salud de la democracia. La caracterizan dos momentos: el básico, elaborado por la Registraduría, sobre los puestos de votación, jurados, tarjeta electoral, tiempos de las elecciones, entre otros; el complejo, realizado, en primer lugar, por los medios, que consiste en el análisis de los temas electorales y la revisión detallada de los programas de gobierno. Este último es el que cualifica la toma de decisiones y permite que las personas voten de manera informada.

Otras consideraciones que se encontraron fueron que la pedagogía electoral tiene como finalidad brindar información oportuna para que el ciudadano contraste y tome decisiones mejor informado, es decir, que se den las condiciones para el voto informado.

\section{Discusión}

Teniendo en cuenta los hallazgos obtenidos con la aplicación de los instrumentos y la triangulación de estos se puede inferir que el periódico regional La Patria cumplió un rol orientador en las pasadas elecciones locales realizadas en octubre de 2019. Para lograrlo, se encontró con un vínculo técnico-periodístico basado en el equilibrio informativo en las publicaciones del periódico sobre el proceso electoral. Así lo corroboró el jefe de Redacción (comunicación personal, noviembre 21, 2019) al indicar que se trató a los aspirantes con equidad informativa, es decir, que en el discurso narrativo de las publicaciones los candidatos tuvieron igual presencia en el proceso informativo. Esto se materializó, especialmente, en los tres perfiles a la Gobernación (Gutiérrez, 2019, octubre 22; Galván, 2019, octubre 27b) y en los cuatro a la Alcaldía, al igual que en los especiales de análisis de los planes de gobierno (Gutiérrez, 2019, octubre 15; Galván, 2019, octubre 27a; Gómez, 2019, octubre 27; Gutiérrez, 2019, octubre 27). Además, la percepción de los lectores encuestados sobre este equilibrio informativo fue 
favorable, porque destacaron a La Patria como un medio preciso durante el cubrimiento periodístico de las elecciones de octubre 2019.

No obstante, dos expertos (Experto 1, comunicación personal, abril 28, 2020; Experto 5, comunicación personal, mayo 8, 2020), consideran que todavía faltan algunos elementos para que se dé dicho equilibrio informativo, como ampliar el cubrimiento a todo el departamento de Caldas y publicar más información sobre los candidatos a las corporaciones y los partidos minoritarios. Por tanto, para que sea efectivo el rol orientador es necesario que haya un tratamiento informativo riguroso, equilibrado y neutral. Asimismo, en estos espacios de información electoral faltó mayor profundidad en la cubertura entre quienes aspiraron a las curules de las Corporaciones -Asamblea de Caldas y Concejo de Manizales- y las Alcaldías de los demás municipios del departamento. La razón principal de esta carencia fue la falta de capacidad técnica y humana del periódico para abarcar estos temas. Estas líneas dan pie para afirmar que garantizar dicho equilibrio informativo no es el culmen de la función orientadora, esta debe ir más allá para que se dé un voto informado y razonado, lo cual se logra cuando el periodista o el medio ayudan a conocer los proyectos políticos y promesas de los candidatos y su viabilidad y pertinencia para la ciudadanía.

Frente a los contenidos del periódico regional La Patria, que constituyan funciones orientadoras, se puede inferir que el diario sí publicó información con elementos orientadores durante la campaña electoral y los presentó de tal forma que Ilamaran la atención del lector. Con base en las teorías de la agenda setting (Zunino, 2018; Rodríguez, 2004; Hernández, 2008; Valderrama, 2006; McQuial, 2000) y framing (Mazzoleni, 2010; Muñiz, Saldierna y Marañón, 2018; Entman, 1993), la agenda periodística se confecciona y se encuadra teniendo en cuenta géneros periodísticos, contextos de las noticias, protagonistas de la información, interés adjudicado al tema, fuentes, autoría de los contenidos publicados y temáticas tratadas.

De esta manera, el periódico regional La Patria tuvo dos enfoques de agendas periodísticas: uno programático, que se refiere a los programas de gobierno de los candidatos y sus análisis, las promesas de campañas expuestas en foros y debates, y la hoja de vida de los aspirantes; y uno logístico, que hace referencia a todo el andamiaje del proceso electoral, es decir, cómo se vota, dónde están las urnas, cuáles son los delitos electorales, las actividades de campaña de los aspirantes, la publicidad electoral y las 
funciones de alcalde, gobernador, diputado, concejal y edil. Lo anterior, según los expertos consultados (Experto 2, comunicación personal, mayo 5, 2020; Experto 3, comunicación personal, mayo 2, 2020), son dos formas de hacer pedagogía o de orientar a los lectores en su derecho al ejercicio del voto, siendo el primer enfoque el más relevante, porque les da a los ciudadanos los elementos de juicio para cualificar sus conversaciones y para tomar decisiones electorales informadas. Sin embargo, en las publicaciones prevaleció más el enfoque de los hechos logísticos de las campañas que el programático.

Las temáticas que más tuvieron preponderancia fueron las actividades de campaña o el registro diario de lo que hicieron los candidatos, por encima de temáticas programáticas como perfiles de los candidatos y análisis de planes de gobierno. Esto confirma que hay un déficit del enfoque programático, es decir, el cubrimiento se centró en la lógica de la noticia del registro diario y dejó a un lado el análisis profundo de los acontecimientos que le diera contexto a los ciudadanos con la finalidad de brindar información suficiente y equilibrada para que se realizara una votación razonada en las urnas. Además, el género periodístico más usado en el cubrimiento de las elecciones regionales de octubre de 2019 fue el informativo o noticioso. Esto da cuenta de que la campaña política de los candidatos locales se cubrió a partir del enfoque noticioso, desde el registro de los hechos, revelando el empleo de este género, que es el más básico, lo que confirma el modelo de periodismo colombiano que suele darle más prioridad al género informativo que a otros como el reportaje, los informes especiales o la crónica. Esto es clave para insistir en que la crónica, el reportaje, la entrevista en profundidad, el perfil y el informe de análisis son géneros pendientes en el periodismo regional a la hora de cubrir las elecciones, teniendo en cuenta que estos proporcionan diversas posturas y miradas sobre diferentes visiones del proceso electoral, enriqueciendo la información.

De igual manera, se puede deducir que en este cubrimiento periodístico los candidatos fueron los protagonistas de las notas periodísticas y, además, las fuentes más consultadas. Esto demuestra que el eje central donde gira y se apoya la información noticiosa, lo que determina cómo se aborda el suceso político, fueron los candidatos. La Patria optó por centrar su información en los candidatos y no en los programas de gobierno o propuestas. Hay un esfuerzo de La Patria por mostrar los perfiles de los candidatos, quiénes son, cuáles son sus relaciones políticas y sus financiadores; por verificar 
y confrontar los programas de gobierno, para corroborar si son viables y pertinentes; y por analizar el panorama político de Caldas y Manizales. No obstante, el diario no hace hincapié en temas como la participación de mujeres en el proceso electoral y la financiación de las campañas políticas, y cuáles son las agendas de los ciudadanos y sus necesidades. El cubrimiento se centró en la Gobernación de Caldas y en la Alcaldía de Manizales, y faltó más información sobre Asamblea, Concejos y Juntas Administradoras Locales y sobre los demás municipios del departamento.

La finalidad de la función orientadora o de la pedagogía electoral es que los ciudadanos voten informados. Se entiende como voto razonado y sensato cuando la persona se informa de la mejor manera acerca de qué o a quién se elige, para qué se vota, qué es lo que propone cada candidato. Lo contrario a esto es un voto emotivo o impulsado por sentimientos y afinidades. Por tanto, se infiere, teniendo en cuenta los resultados obtenidos, que hubo contenidos o información orientadora por parte del medio para que se diera un voto informado. Pero esto se matiza con los hallazgos sobre las actividades que motivaron a decidir por uno u otro candidato.

De acuerdo con los resultados de la encuesta (comunicación personal, febrero, 2020), el ejercicio del voto en las urnas y los debates realizados durante la campaña electoral fueron las actividades que más ayudaron a decidirse por uno o por otro aspirante. Esto demuestra que a pesar de que los lectores se informan y reciben contenidos orientadores por el periódico para ejercer su derecho al voto de manera informada, son otras variables las que impulsan la decisión.

\section{Conclusión}

Se puede concluir que el periódico regional La Patria cumplió un rol orientador en las elecciones locales realizadas en octubre de 2019. No obstante, esta función orientadora no se llevó a cabo en su totalidad, faltó más cubrimiento e información sobre las corporaciones de elección popular; más voces que analizaran y confrontaran las promesas de los candidatos; más datos de los perfiles de los candidatos y de sus estructuras políticas y financieras; más fuentes expertas para darle pluralidad al cubrimiento y no quedarse en la voz oficial de la campaña política; más énfasis en los planes de gobierno y propuestas de los candidatos como elementos para lograr una decisión razonada e informada; más contextualización para darle sentido a la información del proceso electoral. 
Esto lleva a reiterar que mientras haya mayor acceso a la información por parte de los ciudadanos y el periódico regional divulgue contenidos analíticos y orientadores habrá ciudadanos que voten informados y con mayor libertad en la elección, lo cual fortalece la democracia. Queda el reto de pasar de un cubrimiento periodístico básico que recurre al género informativo para presentar el sistema electoral, las actividades de campaña y la logística electoral a un cubrimiento programático que use géneros periodísticos más amplios para otorgar a las personas contenidos y análisis sobre la viabilidad y pertinencia de los programas de gobierno.

\section{Referencias bibliográficas}

1. Angarita, Francisco. (2017). Elección racional, voto obligatorio y venta del voto en Colombia. [Tesis inédita de pregrado]. Universidad Católica de Colombia, Bogotá, D. C. https://repository.ucatolica.edu.co/bitstream/10983/14881/1/ ELECCI\%C3\% 93N\%20RACIONAL \%2C \% 20VOTO $\% 20$ OBLIGATORIO $\% 20$ Y $\% 20$ VENTA $\% 20$ DEL $\% 20$ VOTO $\% 20$ EN $\% 20$ COLOMBIA $\% 20 \% 28$ CORREGIDO $\% 29$.pdf

2. Barrero, Fredy (ed.). (2016). Elecciones regionales 2015: los retos de un país en camino hacia la paz. Bogotá, D. C.: Konrad Adenauer Stiftung.

3. Barrios, Alejandra y Hernández, Fabián. (2012). El rol de las campañas electorales y los medios de comunicación en un proceso electoral equitativo. Cuadernos de Papel, 57, pp. 123-145.

4. Bobbio, Norberto. (1997). El futuro de la democracia. México, D. F.: FCE.

5. Colombia. Congreso de la República. Ley Estatutaria 1581. (17 de octubre de 2012). Por la cual se dictan disposiciones generales para la protección de datos personales. http://www.secretariasenado.gov.co/senado/basedoc/ley_1581_2012.html

6. Consejo de Redacción. (2011, septiembre 29). ¿Qué investigar en época electoral? consejoderedaccion.org/webs/traslapista2015/wp-content/ uploads/2015/11/6.2-Qu\%C3\%A9-investigar-en-\%C3\%A9poca-electoral.pdf

7. Chavarri, Pilar y Delgado, Irene (coords.). (2013). Sistemas políticos contemporáneos. Madrid: UNED.

8. Echeverría, Martín. (2017). Sesgo partidista en medios informativos. Una crítica metodológica y propuesta. Comunicación y Sociedad, 30, pp. 217-238. https://doi. org/10.32870/cys.v0i30.6277

9. Entman, Robert. (1993). Framing: Toward a Clasification of a Factured Paradigm. Journal of Communication, 43, pp. 51-58. https://doi.org/10.1111/j.1460-2466.1993. tb01304.x

10. Espinoza, Gladys. (2004). El periodismo regional. El caso del semanario «El Faro del Callao». [Tesis inédita de pregrado]. Universidad Nacional Mayor de San Marcos, Lima. 
11. Fundación para la Libertad de Prensa (FLIP). (2019). Cartografías de la información. Bogotá, D. C.: FLIP.

12. Galván, Yajandra. (2019, octubre 27a). Andrés Felipe Betancourth, académico. La Patria. https://www.lapatria.com/politica/andres-felipe-betancourthacademico-445672

13. Galván, Yajandra. (octubre 27b). Angelo Quintero Palacio, candidato a la Gobernación de Caldas, del agro. La Patria. https://www.lapatria.com/politica/angeloquintero-palacio-candidato-la-gobernacion-de-caldas-del-agro-446536

14. Gómez, Martha Lucía. (2019, octubre 27). Jorge Hernán Mesa, hombre de cifras. La Patria. https://www.lapatria.com/politica/jorge-hernan-mesa-hombre-decifras-445652

15. Gómez, Rubén. (2015). Rutinas y asimetrías en el ejercicio del periodismo desde la periferia. Perspectivas de la Comunicación, 8 (1), pp. 41-56.

16. Grupo de Investigación Área de Periodismo, Facultad de Comunicación, Universidad de La Sabana. (2002). El papel orientador de la prensa en el cubrimiento de las campañas políticas. La deuda de los medios colombianos. Palabra Clave, 6. https://palabraclave.unisabana.edu.co/index.php/palabraclave/article/view/390/529

17. Guerrero, Arturo. (2010). País lejano y silenciado. Autocensura y prácticas periodísticas en el periodismo regional. Bogotá, D. C.: FLIP.

18. Gutiérrez, Diana Lorena. (2019, octubre 15). Muchas ideas, pero ¿cómo lo harán?: análisis de candidatos a la Alcaldía de Manizales en TIC y turismo. La Patria. https://www.lapatria.com/politica/muchas-ideas-pero-como-lo-haran-analisis-decandidatos-la-alcaldia-de-manizales-en-tic-y

19. Gutiérrez, Diana Lorena. (2019, octubre 22). Las apuestas en TIC, medioambiente y turismo de los candidatos a la Gobernación de Caldas. La Patria. https://www.lapatria.com/politica/las-apuestas-en-tic-medioambiente-y-turismo-delos-candidatos-la-gobernacion-de-caldas

20. Gutiérrez, Diana Lorena. (2019, octubre 27). Jorge Hernán Yepes, el gerente. La Patria. https://www.lapatria.com/politica/jorge-hernan-yepes-el-gerente-445671

21. Gutiérrez, José Luis. (2016). El voto: herramienta de la vida democrática. México, D. F.: Instituto Nacional Electoral.

22. Hernández Sampieri, Roberto. (2008). Las elecciones de la prensa. Agenda informativa y calidad periodística. Bogotá, D. C.: Corporación Medios para la Paz.

23. Hernández Sampieri, Roberto. (2014). Metodología de la investigación. México, D. F.: McGraw-Hill.

24. Klein, Darío. (2001). El papel del periodismo de investigación en la sociedad democrática. Razón y Palabra, 22. http://www.razonypalabra.org.mx/anteriores/ n22/22_dklein.html

25. Kovach, Bill y Rosenstiel, Tom. (2003). Los elementos del periodismo. Madrid: Aguilar. 
26. Krippendorff, Klaus. (1990). Metodología de análisis de contenido: teoría y práctica. Barcelona: Paidós.

27. Leyva, Paulina. (2001). Maxwell McCombs: Hay que reinventar el concepto de noticia. Cuadernos de Información, 12. https://dialnet.unirioja.es/descarga/ articulo/2938991.pdf

28. Luna Salas, Vicky. (2007). Medios regionales y agencias de publicidad: El planificador y su decisión al elegir un medio para pautar. [Tesis inédita de maestría]. Universidad Estatal a Distancia, San José.

29. Maigret, Eric. (2005). Sociología de la comunicación y de los medios. México, D. F.: FCE.

30. Mazzoleni, Gianpietro. (2010). La comunicación política. Madrid: Alianza.

31. McQuial, Denis. (2000). Introducción a la teoría de la comunicación de masas. Barcelona: Paidós.

32. Medellín, Pedro. (2014). Determinantes de la participación electoral en las elecciones de Colombia. Bogotá, D. C.: Registraduría Nacional del Estado Civil.

33. Misión de Observación Electoral (MOE). (2016). Elecciones y medios de comunicación. Autoridades locales 2015. Bogotá D. C.: MOE.

34. Misión de Observación Electoral (MOE). (2018). Medios de comunicación, redes sociales y democracia. Elecciones presidenciales y legislativas 2018. Bogotá, D. C.: MOE.

35. Muñiz, Carlos; Saldierna, Alma y Marañón, Felipe. (2018). Framing of Electoral [264 ] Processes. The Stages of the Campaign as a Moderator of the Presence of Political Frames in the News. Palabra Clave, 21 (3), pp. 740-771. https://doi.org/10.5294/ pacla.2018.21.3.5

36. Nohlen, Dieter. (2004). La participación electoral como objeto de estudio. Elecciones, 3, pp. 137-157.

37. Pérez García, Olga Lucía. (2014). Abecé para periodistas. Elecciones 2014 en Colombia. Bogotá, D. C.: Registraduría Nacional del Estado Civil.

38. Pinzón, Cindy; Ocampo, María y Gutiérrez, Liliana. (2015). Tratamiento periodístico de la Responsabilidad Social Empresarial del sector de alimentos en Colombia. Cuadernos.info, 37, pp. 137-150. https://doi.org/10.7764/cdi.37.686

39. Restrepo, Néstor. (2017). La profesionalización de las campañas electorales en Colombia: Elecciones presidenciales 1994-2014. [Tesis inédita de doctorado). Universidad Complutense de Madrid, Madrid.

40. Rodríguez, Alejandro. (2010). Equilibrio informativo y calidad de la información en El Tiempo y El Espectador. Monitoreo a las presidenciales de 2010. Universidad de Bogotá Jorge Tadeo Lozano. http://avalon.utadeo.edu.co/comunidades/observatorios/ comunicacion/moe/pdf/informe_central.pdf

41. Rodríguez, Raquel. (2004). Teoría de la Agenda Setting, aplicación a la enseñanza universitaria. Alicante: Observatorio Europeo de Tendencias Sociales. 
42. Ronderos, María y Moreno, María. (2006). Cómo cubrir elecciones. Manual para periodistas. Bogotá, D. C.: Fundación Friedrich Ebert en Colombia.

43. Sartori, Giovanni. (1991). Teoría de la democracia, el debate contemporáneo. Tomo 1. México, D. F.: Alianza.

44. Suárez, Enrique. (2005). La verdadera democracia. Las 21 características indispensables. Estudios Políticos, 8 (4), pp. 19-41.

45. Torres, Ana Patricia. (2013). Abstención electoral en Colombia. Desafección política, violencia política y conflicto armado. [Tesis inédita de maestría]. Universidad Complutense de Madrid, Madrid.

46. Valderrama, Jairo. (2006). Medios masivos y función orientadora en las campañas electorales. Palabra Clave, 9 (2), pp. 29-41.

47. Zunino, Esteban. (2018). Agenda Setting: cincuenta años de investigación en comunicación. Intersecciones en Comunicación, 1 (12), pp. 187-210. 